\title{
Generalized Projectively Symmetric Spaces
}

\author{
Dariush Latifi $^{1}$ and Asadollah Razavi ${ }^{2}$ \\ ${ }^{1}$ Department of Mathematics, University of Mohaghegh Ardabili, P.O. Box 56199-11367, Ardabil, Iran \\ ${ }^{2}$ Faculty of Mathematics and Computer Science, Amirkabir University of Technology, 424 Hafez Avenue, \\ P.O. Box 15875-4413, Tehran, Iran
}

Correspondence should be addressed to Dariush Latifi; dlatifi@gmail.com

Received 30 October 2012; Accepted 1 January 2013

Academic Editor: Salvador Hernandez

Copyright (C) 2013 D. Latifi and A. Razavi. This is an open access article distributed under the Creative Commons Attribution License, which permits unrestricted use, distribution, and reproduction in any medium, provided the original work is properly cited.

We study generalized projectively symmetric spaces. We first study some geometric properties of projectively symmetric spaces and prove that any such space is projectively homogeneous and under certain conditions the projective curvature tensor vanishes. Then we prove that given any regular projective s-space $(M, \nabla)$, there exists a projectively related connection $\bar{\nabla}$, such that $(M, \bar{\nabla})$ is an affine s-manifold.

\section{Introduction}

Affine and Riemannian s-manifolds were first defined in [1] following the introduction of generalized Riemannian symmetric spaces in [2]. They form a more general class than the symmetric spaces of E. Cartan. More details about generalized symmetric spaces can be found in the monograph [3]. Let $M$ be a connected manifold with an affine connection $\nabla$, and let $A(M, \nabla)$ be the Lie transformation group of all affine transformation of $M$. An affine transformation $s_{x}$ will be called an affine symmetry at a point $x$ if $x$ is an isolated fixed point of $s_{x}$. An affine manifold $(M, \nabla)$ will be called an affine s-manifold if there is a differentiable mapping $s: M \rightarrow$ $A(M, \nabla)$, such that for each $x \in M, s_{x}$ is an affine symmetry at $x$.

In [4] Podestà introduced the notion of a projectively symmetric space in the following sense. Let $(M, \nabla)$ be a connected $C^{\infty}$ manifold with an affine torsion free connection $\nabla$ on its tangent bundle; $(M, \nabla)$ is said to be projectively symmetric if for every point $x$ of $M$ there is an involutive projective transformation $s_{x}$ of $M$ fixing $x$ and whose differential at $x$ is $-\mathrm{Id}$. The assignment of a symmetry $s_{x}$ at each point $x$ of $M$ can be viewed as a map $s: M \rightarrow P(M, \nabla)$, and $P(M, \nabla)$ can be topologised, so that it is a Lie transformation group. In the above definition, however, no further assumption on $s$ is made; even continuity is not assumed.
In this paper we define and state prerequisite results on projective structures and define projective symmetric spaces due toPodestà. Then we generalize them to define projective s-manifolds as manifolds together with more general symmetries and consider the cases where they are essential or inessential. A projective s-manifold is called inessential if it is projectively equivalent to an affine s-manifold and essential otherwise. We prove that these spaces are naturally homogeneous, and moreover under certain conditions the projective curvature tensor vanishes. Later we define regular projective s-manifolds and prove that they are inessential.

\section{Preliminaries}

Let $M$ be a connected real $C^{\infty}$ manifold whose tangent bundle TM is endowed with an affine torsion free connection $\nabla$. We recall that a diffeomorphism $s$ of $M$ is said to be projective transformation if $s$ maps geodesics into geodesics when the parametrization is disregarded [5]; equivalently $s$ is projective if the pull back $s^{*} \nabla$ of the connection is projectively related to $\nabla$, that is, if there exists a global 1 -form $\pi$ on $M$, such that

$$
s^{*} \nabla_{X} Y=\nabla_{X} Y+\pi(X) Y+\pi(Y) X \quad \forall X, Y \in \chi(M) .
$$

If the form $\pi$ vanishes identically on $M$, then $s$ is said to be an affine transformation. 
Definition 1. $(M, \nabla)$ is said to be projectively symmetric if for every point $x$ in $M$ there exists a projective transformation $s_{x}$ with the following properties:

(a) $s_{x}(x)=x$ and $x$ is an isolated fixed point of $s_{x}$.

(b) $s_{x}$ is involutive.

It is easy to see that conditions (a) and (b) imply $\left(d s_{x}\right)_{x}=$ -Id. Moreover we recall that a projective transformation is determined if we fix its value at a point, its differential, and its second jet at this point [6], hence a symmetry at $x$ in $M$ is not uniquely determined in general by the condition (a) and (b).

Affine symmetric spaces are affine homogeneous, but in general projectively symmetric spaces are not projective homogeneous; for more detail and examples see $[4,7]$, but if following Ledger and Obata define the case of a differentiable distribution of projective symmetries in an affine manifold, then this happens.

Let $(M, g)$ be a connected Riemannian manifold. An isometry $s_{x}$ of $(M, g)$ for which $x \in M$ is an isolated fixed point will be called a Riemannian symmetry of $M$ at $x$. Clearly, if $s_{x}$ is a symmetry of $(M, g)$ at $x$, then the tangent map $S_{x}=\left(d s_{x}\right)_{x}$ is an orthogonal transformation of $T_{x} M$ having no fixed vectors (with the exception of 0 ). An sstructure on $(M, g)$ is a family $\left\{s_{x} \mid x \in M\right\}$ of symmetries of $(M, g)$.

A Riemannian s-manifold is a Riemannian manifold $(M, g)$ together with a map $s: M \rightarrow I(M, g)$, such that for each $x \in M$ the image $s_{x}$ is a Riemannian symmetry at $x$.

For any affine manifold $(M, \nabla)$ let $A(M, \nabla)$ denote the Lie group of all affine transformation of $(M, \nabla)$. An affine transformation $s_{x} \in A(M, \nabla)$ for which $x \in M$ is an isolated fixed point will be called an affine symmetry at $x$. An affine s-manifold is an affine manifold $(M, \nabla)$ together with a differentiable mapping $s: M \rightarrow A(M, \nabla)$, such that for each $x \in M$, the image $s_{x}$ is an affine symmetry at $x$.

Let $M$ be an affine s-manifold. Since $s: M \rightarrow A(M, \nabla)$ is assumed to be differentiable, the tensor field $S$ of type $(1,1)$ defined by $S_{x}=\left(d s_{x}\right)_{x}$ for each $x \in M$ is differentiable. The tensor field $S$ is defined similarly for a Riemannian smanifold, although it may not be smooth. For either affine or Riemannian s-manifolds we call $S$ the symmetry tensor field.

Following [3] an s-structure $\left\{s_{x}\right\}$ is called regular if for every pair of points $x, y \in M$ as follows:

$$
s_{x} \circ s_{y}=s_{z} \circ s_{x}, \quad \text { where } z=s_{x}(y) .
$$

\section{Projective s-Space}

Let $M$ be a connected manifold with an affine connection $\nabla$, and let $P(M, \nabla)$ bethe group of all projective transformations of $M$.

Definition 2. A projective transformation $s_{x}$ will be called a projective symmetry or simply a symmetry at the point $x$, if $x$ is an isolated fixed point of $s_{x}$ and $\left(d s_{x}\right)_{x}=S$ does not leave any nonzero vector fixed.

Definition 3. A connected affine manifold $(M, \nabla)$ will be called a projective s-manifold or simply ps-manifold if for each $x \in M$ there is a projective symmetry $s_{x}$, such that the mapping $s: M \rightarrow P(M, \nabla), x \mapsto s_{x}$ is smooth.

A symmetry $s_{x}$ will be called a symmetry of order $k$ at $x$, if there exist a positive integer $k$, such that $s_{x}^{k}=\mathrm{Id}$, and $M$ will be called ps-manifold of order $k$, if $k$ is the least positive number such that each symmetry is of order $k$. Evidently every ps-manifold of order 2 is a projective symmetric space.

Lemma 4. Let $G$ be a topological transformation group acting on a connected topological space $M$, iffor each point $x$ in $M$, the $G$-orbit of $x$ contains a neighborhood of $x$, then $G$ is transitive on $M$.

Proof. Since $G$ is transitive on each orbit, for each $x$ the $G$ orbit $G(x)$ of $x$ is open by our assumption. The complement $C(x)$ of $G(x)$ in $M$ is also open, being a union of orbits. Thus $G(x)$ is open and closed. It is nonempty and therefore coincides with the connected space $M$, thus $G$ is transitive.

Theorem 5. If $M$ is a ps-manifold, then $P(M, \nabla)$ is transitive on $M$.

Proof. We fix a point $x_{0} \in M$ and consider the $C^{\infty}$ map $h$ : $M \rightarrow M$ given by $h(x)=s_{x}\left(x_{0}\right)$; since $s_{x}(x)=x$ for every $x$ in $M$, the differential $(d h)_{x_{0}}$ of $h$ at the point $x_{0}$ is given by $(d h)_{x_{0}}=I-S_{x_{0}}$, where $S_{x_{0}}$ is the differential of $s_{x_{0}}$ at $x_{0}$. $(d h)_{x_{0}}$ is nonsingular because no eigenvalue of $S_{x_{0}}$ is equal to 1. Hence $h$ is a diffeomorphism on some neighborhood $W$ of $x_{0}$ in $M$, and $h(W)$ is a neighborhood of $x_{0}$ contained in the $P(M, \nabla)$-orbit $P(M, \nabla)_{x_{0}}$ of $x_{0}$, therefore from the above lemma $P(M, \nabla)$ is transitive.

Definition 6. Let $M$ be a ps-manifold; since $s: M \rightarrow$ $P(M, \nabla)$ is assumed to be differentiable, the tensor field $S$ of type $(1,1)$ defined by $S_{x}=\left(d s_{x}\right)_{x}$ is differentiable, we call $S$ the symmetry tensor field.

Lemma 7. If $s_{x}$ is a projective symmetry of $(M, \nabla)$ then there exists a connection $\bar{\nabla}$ projectively equivalent with $\nabla$ which is $s_{x}$-invariant.

Proof. Since $s_{x}$ is a projective symmetry of $(M, \nabla)$ then there is a 1 -form $\alpha$ on $M$, such that

$$
\left(s_{x}^{*} \nabla\right)_{X} Y=\nabla_{X} Y+\alpha(X) Y+\alpha(Y) X .
$$

We are looking for a connection $\bar{\nabla}$ with the following properties:

$$
\bar{\nabla}_{X} Y=\nabla_{X} Y+\pi(X) Y+\pi(Y) X
$$

As $\bar{\nabla}$ should be $s_{x}$-invariant we need

$$
\left(s_{x}^{*} \bar{\nabla}\right)_{X} Y=\bar{\nabla}_{X} Y
$$


that is, $s_{x}$ is an affine transformation of $(M, \bar{\nabla})$. We have

$$
\begin{aligned}
\left(s_{x}^{*} \bar{\nabla}\right)_{X} Y= & s_{x_{*}}^{-1} \bar{\nabla}_{s_{x_{*}} X} s_{x_{*}} Y \\
= & s_{x_{*}}^{-1}\left(\nabla_{s_{x_{*}} X} s_{x_{*}} Y+\pi\left(s_{x_{*}} X\right) s_{x_{*}} Y\right. \\
& \left.\quad+\pi\left(s_{x_{*}} Y\right) s_{x_{*}} X\right) \\
= & \left(s_{X}^{*} \nabla\right)_{X} Y+\pi\left(s_{x_{*}} X\right) Y+\pi\left(s_{x_{*}} Y\right) X .
\end{aligned}
$$

It follows from (3)

$$
\begin{aligned}
\left(s_{x}^{*} \bar{\nabla}\right)_{X} Y= & \nabla_{X} Y+\alpha(X) Y+\alpha(Y) X \\
& +\pi\left(s_{x_{*}} X\right) Y+\pi\left(s_{x_{*}} Y\right) X .
\end{aligned}
$$

From (5) we have

$$
\begin{aligned}
\nabla_{X} Y & +\alpha(X) Y+\alpha(Y) X+\pi\left(s_{x_{*}} X\right) Y+\pi\left(s_{x_{*}} Y\right) X \\
& =\nabla_{X} Y+\pi(X) Y+\pi(Y) X
\end{aligned}
$$

thus it is enough to have for every vector field $Z$ as follows:

$$
\alpha(Z)+\pi\left(s_{x_{*}} Z\right)=\pi(Z)
$$

which is equivalent to

$$
\pi\left(Z-s_{x_{*}} Z\right)=\alpha(Z)
$$

or simply

$$
\pi \circ\left(I-s_{x_{*}}\right) Z=\alpha(Z)
$$

since $s_{x}$ is symmetry, then $I-s_{x}$ is invertible; hence we obtain

$$
\pi=\alpha \circ\left(I-s_{x_{*}}\right)^{-1}
$$

thus if we choose $\pi$ as (12), then (4) and (5) are true, and $\bar{\nabla}$ is the required connection.

So it would be convenient to introduce the following definition for connection $\bar{\nabla}$ and 1-form $\pi=\alpha \circ\left(I-s_{x_{*}}\right)^{-1}$.

Definition 8 . Let $(M, \nabla)$ be a ps-manifold, and let $s_{x}$ be the projective symmetry at the point $x$. Then we call the associate connection $\bar{\nabla}$ the fundamental connection of $s_{x}$. Also the 1form

$$
\pi=\alpha \circ\left(I-s_{x_{*}}\right)^{-1}
$$

will be called the fundamental 1-form of $s_{x}$, where $\alpha$ is the 1 -form on $M$, such that

$$
s^{*} \nabla_{X} Y=\nabla_{X} Y+\pi(X) Y+\pi(Y) X \quad \forall X, Y \in \chi(M) .
$$

Definition 9. The projective curvature tensor of $(M, \nabla)$ is defined as follows $[5,8]$ :

$$
W_{j k l}^{i}=\Pi_{j k l}^{i}-\frac{1}{n-1}\left(\delta_{k}^{i} \Pi_{j l}-\delta_{l}^{i} \Pi_{j k}\right),
$$

where

$$
\begin{gathered}
\Pi_{j k}^{i}=\Gamma_{j k}^{i}-\frac{2}{n+1} \delta_{(j}^{i} \Gamma_{k) l}^{l}, \\
\Pi_{j k l}^{i}=\partial_{k} \Pi_{j l}^{i}-\partial_{l} \Pi_{j k}^{i}+\Pi_{j l}^{h} \Pi_{h k}^{i}-\Pi_{j k}^{h} \Pi_{h l}^{i}, \quad \Pi_{j k}=\Pi_{j h k}^{h} .
\end{gathered}
$$

The projective curvature tensor $W$ is invariant with respect to projective transformations $[5,8]$.

Theorem 10. In a ps-manifold $(M, \nabla)$, let $s_{x}$ be a symmetry, and let $\bar{\nabla}$ be the fundamental connection of $s_{x}$, if $\bar{\nabla} S=0$; that is, $\left(\left(\nabla_{X} S\right)(Y)=\pi(Y) S(X)-\pi(S(Y)) X\right)$, then $(\bar{\nabla} W)_{x}=0$.

Proof. Let $s: M \rightarrow P(M, \nabla)$ be the $p s$-structure and $\bar{\nabla} S=0$. Let $X, Y, Z \in T_{x} M$ be tangent vectors, and let $\omega \in T_{x}^{*} M$ be a covector at $x \in M$. By parallel translation along each geodesics through $x, X, Y, Z$, and $\omega$ can be extended to local vector fields $\widetilde{X}, \widetilde{Y}, \widetilde{Z}$, and $\widetilde{\omega}$ with vanishing covariant derivative with respect to $\bar{\nabla}$ at $x$. Because $S$ is parallel, the local vector fields $S \widetilde{X}, S \widetilde{Y}, S \widetilde{Z}$, and $S^{*} \widetilde{\omega}$ have also vanishing covariant derivative at $x$. (Here $S^{*}$ denotes the transpose map to $S$.) As $W$ is invariant with respect to the projective transformation $s_{p}, p \in M$, we have

$$
W\left(S^{*} \widetilde{\omega}, \widetilde{X}, \widetilde{Y}, \widetilde{Z}\right)=W(\widetilde{\omega}, S \widetilde{X}, S \widetilde{Y}, S \widetilde{Z}) .
$$

Now we show that $\bar{\nabla} W\left(S^{*} \widetilde{\omega}, \widetilde{X}, \tilde{Y}, \widetilde{Z}, U\right)$ and $\bar{\nabla} W(\widetilde{\omega}, S \widetilde{X}$, $S \widetilde{Y}, S \widetilde{Z}, S U)$ are equal at $x$. These are equal if and only if $\left(S^{*} \omega\right)\left(\bar{\nabla}_{U} W(\widetilde{X}, \widetilde{Y}, \widetilde{Z})\right)$ and $\omega\left(\bar{\nabla}_{S U} W(S \widetilde{X}, S \widetilde{Y}, S \widetilde{Z})\right)$ are equal, which follows from the assumption on $\bar{\nabla}$. That is

$$
\bar{\nabla} W\left(S_{x}^{*} \omega, X, Y, Z, U\right)=\bar{\nabla} W\left(\omega, S_{x} X, S_{x} Y, S_{x} Z, S_{x} U\right)
$$

or

$$
\bar{\nabla} W(\omega, X, Y, Z, U)=\bar{\nabla} W\left(S^{*-1} \omega, S X, S Y, S Z, S U\right) .
$$

Differentiating covariantly (17) with respect to $\bar{\nabla}$ in the direction of $S U$ at $x$ and using (19) we get

$$
\bar{\nabla} W(\omega, X, Y, Z, S U)=\bar{\nabla} W(\omega, X, Y, Z, U),
$$

thus

$$
(\bar{\nabla} W)_{x}(\omega, X, Y, Z,(I-S) U)=0,
$$

for all $X, Y, Z, U \in T_{x} M$ and $\omega \in T_{x}^{*} M$, and because $(I-S)_{x}$ is a nonsingular transformation we obtain

$$
(\bar{\nabla} W)_{x}=0
$$

Theorem 11. Let $(M, \nabla)$ be a ps-manifold of dimension $n>$ 2 ; if there exist two different projective symmetries $\sigma_{1}, \sigma_{2}$ at a point $q$ of $M$, such that $\sigma_{1 * q}=\sigma_{2 * q}$ and $\bar{\nabla} S=0$, where $\bar{\nabla}$ is the fundamental connection corresponding to $\sigma_{1}$, then the projective curvature tensor $W$; vanishes that is, $M$ is projectively flat. 
Proof. By a similar method used in Proposition 1.1 of [7] the proof follows from Lemma 7 and Theorem 10.

Corollary 12. If $(M, \nabla)$ is a ps-manifold of order 2, and two different projective symmetry $\sigma_{1}, \sigma_{2}$ can be defined at a point $q$, then $M$ is projectively flat.

Proof. It is evident from the fact that $\sigma_{1 * q}=\sigma_{2 * q}$.

Proposition 13. Let $(M, \nabla)$ be ps-manifold, such that at every point $x$ of $M$ the projective symmetry is uniquely determined. Then the linear isotropy representation $\rho: P(M, \nabla)_{x} \rightarrow$ $G L(n, R)$ is faithful for every $x \in M$.

Proof. Since $s_{x}$ and $s_{x}^{-1}$ both are projective symmetry at $x$, then we have $s_{x}=s_{x}^{-1}$; that is, $s_{x}^{2}=\operatorname{Id}$, thus $(M, \nabla)$ is a ps-manifold of order 2. Now, our assertion follows from Theorem 1.1 of [7].

\section{Regular Projective s-Space}

Definition 14. A $p s$-manifold $(M, \nabla)$ is called regular $p s$ manifold or simply $r p s$-manifold if for all $p, q \in M, s_{p} \circ s_{q}=$ $s_{z} \circ s_{p}$, where $z=s_{p}(q)$.

Lemma 15. Let $(M, \nabla)$ be a regular ps-manifold, then the $(1,1)$ tensor field $S$ is invariant under all symmetries $s_{x}$; that is

$$
d s_{x}(S X)=S\left(d s_{x} X\right)
$$

for all $X \in \chi(M)$.

Proof. Since $M$ is regular ps-manifold, then for all $X \in T_{y} M$ we have $d\left(s_{x} \circ s_{y}\right) X=d\left(s_{z} \circ s_{x}\right) X$ and so $d s_{x}(S X)=S_{z}\left(d s_{x} X\right)$. Thus $S$ is $s_{x}$ invariant for all $s_{x}$.

Lemma 16. Let $(M, \nabla)$ be a connected ps-manifold, such that at every point $x$ of $M$ the projective symmetry is uniquely determined, then $(M, \nabla)$ is rps-manifold.

Proof. Suppose $p, q \in M$ and $z=s_{p}(q)$; then from the uniqueness of the projective symmetry, we have $s_{p} \circ s_{q}=s_{z} \circ s_{p}$, so $(M, \nabla)$ is regular ps-manifold.

Remark 17. A general question is to find condition under which, given a ps-manifold $(M, \nabla)$, there exists a projectively related connection $\bar{\nabla}$, such that $(M, \bar{\nabla})$ is an affine s-manifold; we shall call such spaces inessential ps-manifold and essential otherwise.

Definition 18. A ps-manifold $(M, \nabla)$ is called inessential psmanifold if there exists a projectively related connection $\bar{\nabla}$ such that $(M, \bar{\nabla})$ is an affine s-manifold.

Let us denote by $\Phi_{h}$ the 1-form corresponding to an element $h$ of $P(M, \nabla)$. We want to see when $(M, \nabla)$ is inessential, in order to show that $(M, \nabla)$ is inessential, we must find a connection $\bar{\nabla}$ which is projectively related to $\nabla$ and is invariant under all symmetries. Let $s_{q}$ be a symmetry at $q$, we must find a one-form $\pi$, such that

$$
\bar{\nabla}_{X} Y=\nabla_{X} Y+\pi(X) Y+\pi(Y) X .
$$

As $s_{q}$ is a projective transformation for $(M, \nabla)$ and leaves the connection $\bar{\nabla}$ invariant, we find that

$$
\Phi_{s_{q}}(X)+\pi\left(s_{q_{*}} X\right)=\pi(X) \quad \forall X \in \chi(M)
$$

and hence at $q$ we have

$$
\pi_{\mid q}\left(I-\left(s_{q}\right)_{* q}\right) X=\Phi_{s_{q}}(X) .
$$

So we define a 1-form $\pi$ through the following formula:

$$
\pi_{\mid x}(X)=\Phi_{s_{x \mid x}} \circ\left(I-\left(s_{x}\right)_{* x}\right)^{-1} X \quad \forall X \in T_{x} M .
$$

Theorem 19. Let $(M, \nabla)$ be an rps-manifold, then $(M, \nabla)$ is inessential.

Proof. We define a torsion free affine connection $\bar{\nabla}$ projectively related to $\nabla$ through the fundamental 1-form of $s_{x}, \pi$ as follows:

$$
\pi_{\mid x}(X)=\Phi_{s_{x \mid x}} \circ\left(I-\left(s_{x}\right)_{* x}\right)^{-1} X \quad \forall X \in T_{x} M
$$

and prove that the connection $\bar{\nabla}$ is invariant under all the symmetries of $M$.

Let $s_{q}$ be a symmetry at $q$ of $M$, the condition that $\bar{\nabla}$ is invariant under $s_{q}$ is equivalent to

$$
\pi(X)-\pi\left(s_{q^{*}} X\right)=\Phi_{s_{q}}(X) .
$$

We verify (29) at a point $p$ of $M$, we have to prove that by (28)

$$
\Phi_{s_{p \mid p}} \circ\left(I-\left(s_{p}\right)_{* p}\right)^{-1} X-\pi_{s_{q}(p)}\left(s_{\left.q *\right|_{p}} X\right)=\Phi_{s_{\left.q\right|_{p}}}(X)
$$

so if we put $z=s_{q}(p),(30)$ reduces to

$$
\begin{aligned}
& \Phi_{s_{p \mid p}} \circ\left(I-\left(s_{p}\right)_{* p}\right)^{-1} X-\Phi_{s_{z \mid z}} \circ\left(I-\left(s_{z}\right)_{* z}\right)^{-1}\left(s_{q * \mid p} X\right) \\
& \quad=\Phi_{s_{q \mid p}}(X) .
\end{aligned}
$$

But since $s_{q} \circ s_{p}=s_{z} \circ s_{q}$, we have

$$
\Phi_{s_{p}}(Y)+\Phi_{s_{q}}\left(s_{p *} Y\right)=\Phi_{s_{q}}(Y)+\Phi_{s_{z}}\left(s_{q *} Y\right) \text {. }
$$

Now evaluate (32) at $p$, and let $Y=\left(I-\left(s_{p}\right)_{* p}\right)^{-1} X$; then as $s_{q} \circ s_{p}=s_{z} \circ s_{q}$, we have (31), and we are done.

Remark 20. The authors have studied Finsler homogeneous and symmetric spaces [9]; recently Habibi and the second author generalized them to Finsler s-manifolds and weakly Finsler symmetric spaces $[10,11]$. Therefore these concepts can be mixed and find more generalizations which will be the content of other papers. 


\section{Acknowledgment}

The authors would like to thank the anonymous referees for their suggestions and comments, which helped in improving the paper.

\section{References}

[1] A. J. Ledger and M. Obata, "Affine and Riemannian smanifolds," Journal of Differential Geometry, vol. 2, pp. 451-459, 1968.

[2] A. J. Ledger, "Espaces de Riemann symétriques généralisés," Comptes Rendus de l'Académie des Sciences, vol. 264, pp. A947A948, 1967.

[3] O. Kowalski, Generalized Symmetric Spaces, vol. 805 of Lecture Notes in Mathematics, Springer, Berlin, Germany, 1980.

[4] F. Podestà, "Projectively symmetric spaces," Annali di Matematica Pura ed Applicata. Serie Quarta, vol. 154, pp. 371-383, 1989.

[5] A. V. Aminova, "Projective transformations of pseudoRiemannian manifolds," Journal of Mathematical Sciences, vol. 113, no. 3, pp. 367-470, 2003.

[6] S. Kobayashi, Transformation Groups in Differential Geometry, Springer, Berlin, Germany, 1980.

[7] F. Podestà, "A class of symmetric spaces," Bulletin de la Société Mathématique de France, vol. 117, no. 3, pp. 343-360, 1989.

[8] L. P. Eisenhart, Non-Riemannian Geometry, vol. 8 of American Mathematical Society Colloquium Publications, American Mathematical Society, 1927.

[9] D. Latifi and A. Razavi, "On homogeneous Finsler spaces," Reports on Mathematical Physics, vol. 57, no. 3, pp. 357-366, 2006.

[10] P. Habibi and A. Razavi, "On generalized symmetric Finsler spaces," Geometriae Dedicata, vol. 149, pp. 121-127, 2010.

[11] P. Habibi and A. Razavi, "On weakly symmetric Finsler spaces," Journal of Geometry and Physics, vol. 60, no. 4, pp. 570-573, 2010. 


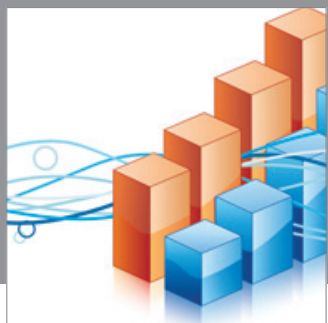

Advances in

Operations Research

mansans

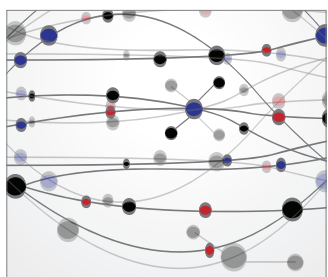

The Scientific World Journal
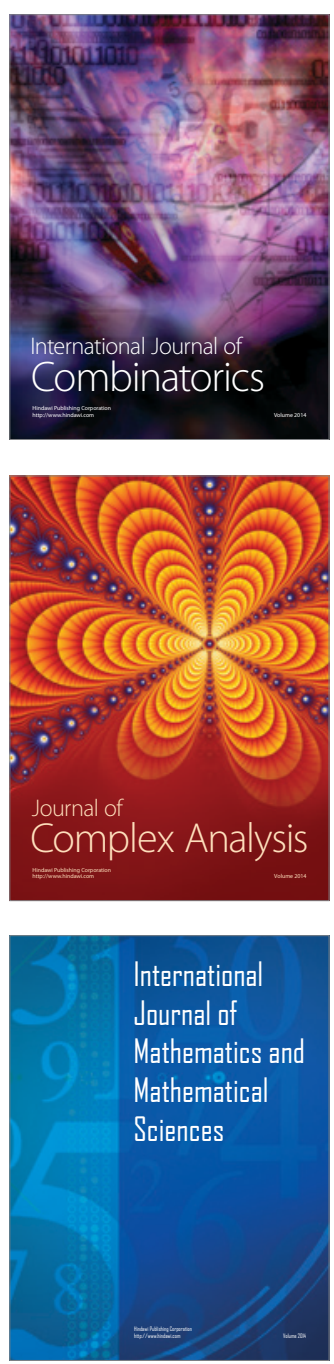
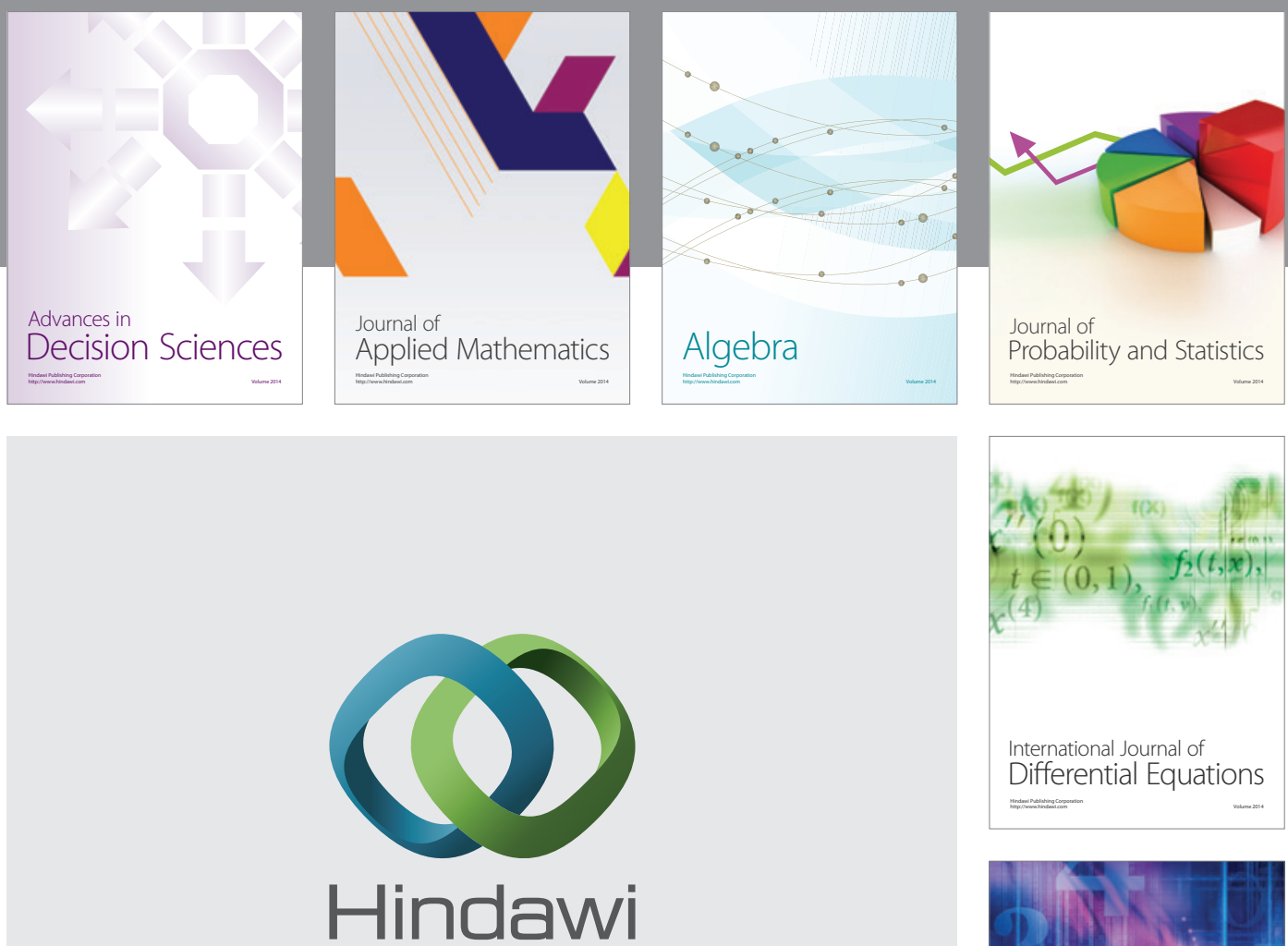

Submit your manuscripts at http://www.hindawi.com
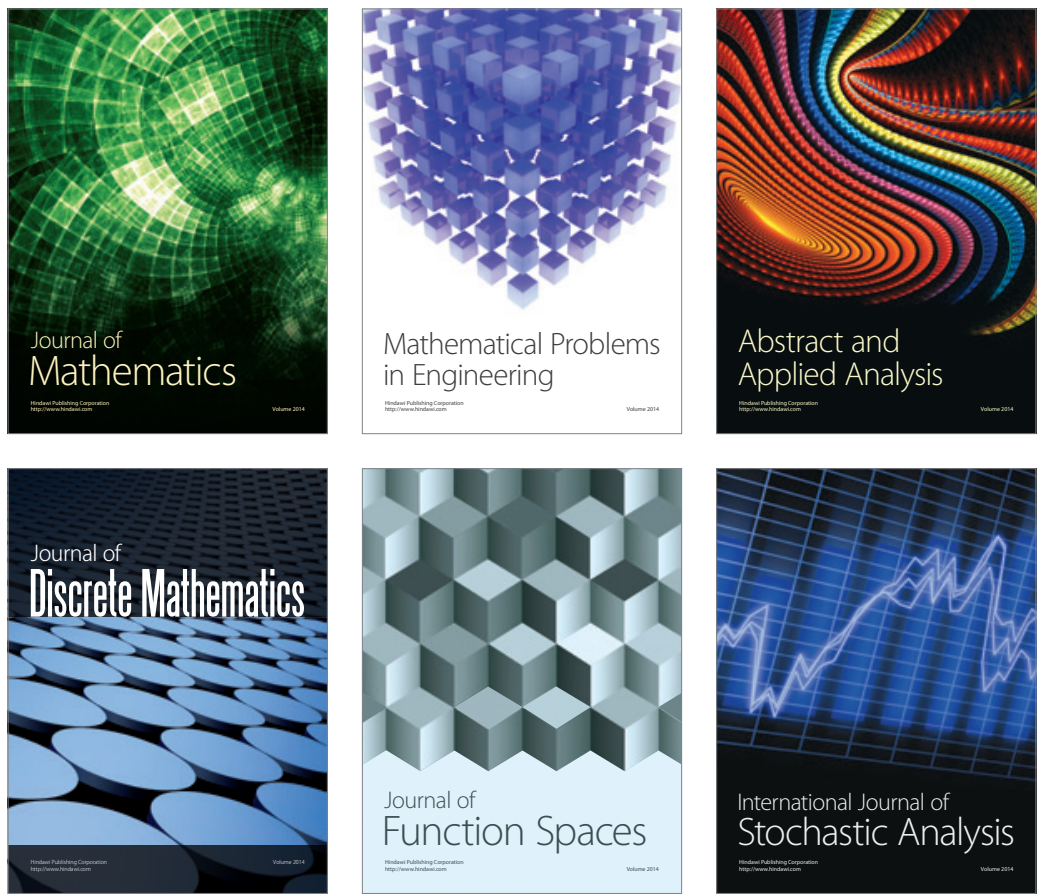

Journal of

Function Spaces

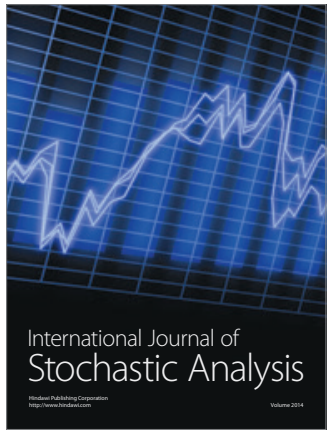

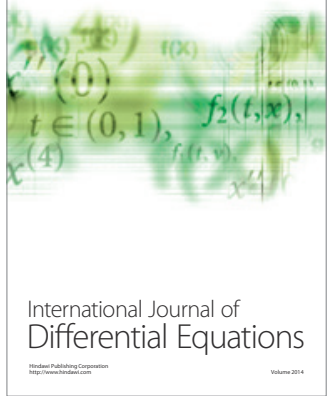
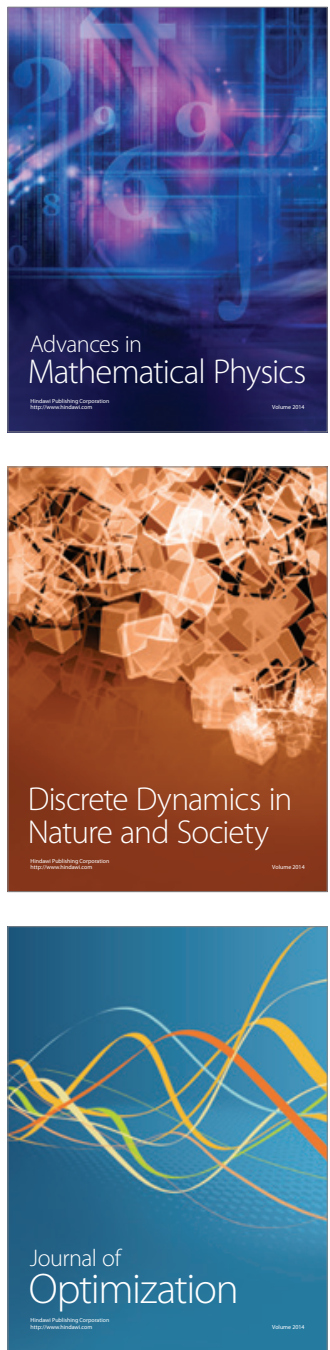